\title{
Medical Radiation Physics
}

National Cancer Institute

\section{Source}

National Cancer Institute. Medical Radiation Physics. NCI Thesaurus. Code C18870.

The study of ionizing radiation and its effects on matter as it pertains to clinical use and practice. 